\title{
Profils Épidémiologiques, Cliniques et Thérapeutiques de la Sténose Urétrale de L'homme à l'Hôpital National de Zinder (HNZ), Niger
}

Maazou Halidou, (Urologue, MD, MS)

Harissou Adamou, (Chirurgie générale, MD, MS)

Université de Zinder, Faculté des sciences de la santé, Département de chirurgie et spécialités chirurgicales, Hôpital National de Zinder, Niger

Diongolé Hassane, (Néphrologie, MD, MS)

Université de Zinder, Faculté des sciences de la santé, Département de Médecine et spécialités médicales, Hôpital National de Zinder, Niger

Mahamadou Doutchi, (Infectiologue, MD, MS)

Université de Zinder, Faculté des sciences de la santé, Service d'infectiologie, Hôpital National de Zinder, Niger

Ibrahim Amadou Magagi, (Chirurgie générale, MD, MS) Université de Zinder, Faculté des sciences de la santé, Département de chirurgie et spécialités chirurgicales, Hôpital National de Zinder, Niger

Ousseini Adakal, (Chirurgie générale, MD, MS) Amadou Roua, (Urologue, MD, MS)

Université Dan Dicko Dan Koulodo de Maradi, Faculté des sciences de la santé, Département de chirurgie et spécialités chirurgicales, Hôpital régional de Maradi, Niger

Soumana Djibo, (Urologue, MD, MS)

Soumana Amadou, (Urologue, MD, MS, MCA)

Université Abdou Moumouni de Niamey, Faculté des sciences de la santé, Département de chirurgie et spécialités chirurgicales, Hôpital National de Zinder, Niger

Doi:10.19044/esj.2020.v16n9p103～ＵRL:http://dx.doi.org/10.19044/esj.2020.v16n9p103

Résumé

Objectif : Décrire les aspects épidémiologiques, cliniques et thérapeutiques de la sténose urétrale à L'hôpital national de Zinder (HNZ) NIGER. Patients et méthodes : Il s'agissait d'une étude rétrospective descriptive réalisée dans le service d'Urologie de l'HNZ durant la période de janvier 2016 à Décembre 2019. Nous avons colligé 116 dossiers médicaux des patients, chez qui le diagnostic de rétrécissement urétral était posé et documenté par une urétro-cystographie rétrograde et mictionnelle (UCRM) et 
pris en charge. Résultats : Au cours de cette étude, 116 dossiers étaient retenus sur 964 interventions durant la période soit 8,31\%.L'âge moyen des patients était de 39,53 ans avec des extrêmes de 18 ans et 74 ans. Les principaux motifs de consultations étaient la dysurie $(25,86 \%)$, la pollakiurie $(15,52 \%)$, et la rétention aiguë dans $(17,24 \%)$. L'urétro-cystographie rétrograde et mictionnelle (UCRM) était le principal examen complémentaire du diagnostic. Cet examen avait permis de découvrir, 69,3\% de sténose urétrale au niveau bulbaire, $17,5 \%$ au niveau pénien antérieur, $9,4 \%$ multiples, $3,8 \%$ au niveau du méat urétral. Le traitement avait consisté en une résection anastomose termino-terminale (RATT) au niveau bulbaire dans $57,21 \%(\mathrm{n}=67)$, de dilatations urétrales (DU) aux béniqués dans 19, 01\% $(\mathrm{n}=22)$ des cas, urétroplastie en deux temps selon Johanson dans 11, $52 \%(\mathrm{n}=13)$, de plasties par greffe de peau glabre de la crête iliaque dans $8,42 \%(\mathrm{n}=10)$, et une méatotomie $3,45 \%(\mathrm{n}=4)$. Après un recul de 21 mois, les anastomoses (RATT) étaient jugées bonnes pour $61,19 \%$ (41/67). Les échecs étaient enregistrés dans $16,42 \%(\mathrm{n}=11)$ et $22,39 \%(\mathrm{n}=15)$ étaient perdus de vue. Pour les dilatations aux béniqués à 9 mois de recul, $59 \%(\mathrm{n}=$ 13) étaient évaluées bonnes, et mauvaises dans $41 \%(\mathrm{n}=9)$ des cas. Les plasties avec greffe étaient réussies dans 30\% ( $\mathrm{n}=3)$ des cas, et $70 \%(\mathrm{n}=7)$ étaient des échecs. Les urétroplasties selon Johanson étaient jugées satisfaisantes à six mois de récul dans $23,07 \%$ (3/13) des cas, et non satisfaisantes dans 76,93\% (10/13) des cas.Toutes les méatotomies étaient réussies ( $\mathrm{n}=4)$. Un RPM $\leq 30 \mathrm{ml}$ était enregistré dans $55,17 \%$ des cas et $\geq$ $100 \mathrm{ml}$ dans $31,90 \%$ des cas. Quinze patients soit 12,93 étaient perdus de vue après leur première évaluation. Conclusion : La sténose de l'urètre est une affection fréquente dans les consultations en urologie à l'HNZ. Les patients consultent souvent au stade de complications. L'UCRM est la clé du diagnostic topographique de la lésion. La résection anastomose terminoterminale donne de bons résultats. La maitrise des plasties avec greffe de muqueuse, ou cutanée reste encore un défi dans notre contexte.

Mots Clés : Sténose, Urètre, Urétroplastie, Chirurgie, Zinder 


\section{Epidemiological, Clinical and Therapeutic Profiles of male Urethral Stricture at Zinder National Hospital (HNZ), Niger}

Maazou Halidou, (Urologue, MD, MS)

Harissou Adamou, (Chirurgie générale, MD, MS)

Université de Zinder, Faculté des sciences de la santé, Département de chirurgie et spécialités chirurgicales, Hôpital National de Zinder, Niger

Diongolé Hassane, (Néphrologie, MD, MS)

Université de Zinder, Faculté des sciences de la santé, Département de Médecine et spécialités médicales, Hôpital National de Zinder, Niger.

Mahamadou Doutchi, (Infectiologue, MD, MS)

Université de Zinder, Faculté des sciences de la santé, Service d'infectiologie, Hôpital National de Zinder, Niger

Ibrahim Amadou Magagi, (Chirurgie générale, MD, MS) Université de Zinder, Faculté des sciences de la santé, Département de chirurgie et spécialités chirurgicales, Hôpital National de Zinder, Niger.

Ousseini Adakal, (Chirurgie générale, MD, MS) Amadou Roua, (Urologue, MD, MS)

Université Dan Dicko Dan Koulodo de Maradi, Faculté des sciences de la santé, Département de chirurgie et spécialités chirurgicales, Hôpital régional de Maradi, Niger.

Soumana Djibo, (Urologue, MD, MS)

Soumana Amadou, (Urologue, MD, MS, MCA)

Université Abdou Moumouni de Niamey, Faculté des sciences de la santé, Département de chirurgie et spécialités chirurgicales, Hôpital National de Zinder, Niger

Abstract

Objective: To describe the epidemiological, clinical and therapeutic aspects of urethral stenosis at Zinder National Hospital (ZNH) NIGER. Patients and methods: This was a descriptive retrospective study carried out in the Department of Urology of the ZNH during the period from January 2016 to December 2019. We collected 116 medical records of patients, in whom the diagnosis of narrowed Urethral donation was performed and documented by retrograde and voiding urethro-cystography and managed. Results: During 
this study, 116 files were retained on 964 interventions during the period, ie $8.31 \%$. The average age of the patients was 39.53 years with extremes of 18 and 74 years. The main reasons for consultations were dysuria $(25.86 \%)$, pollakiuria (15.52\%), and acute retention in (17.24\%). Retrograde and voiding urethro-cystography (UC) was the main complementary diagnostic examination. This examination had revealed $69.3 \%$ urethral stenosis at the bulbar level, $17.5 \%$ at the anterior penis level, $9.4 \%$ multiple, $3.8 \%$ at the urethral meatus. The treatment consisted of resection of the end-to-end anastomosis at the bulbar level in $57.21 \%(n=67)$, from urethral dilation to the benign in $19.01 \%(\mathrm{n}=22)$ of the cases, urethroplasty in two stages according to Johanson in $11.52 \%(\mathrm{n}=13)$, of plasties by glabrous skin graft of the iliac crest in $8.42 \%(n=10)$, and a meatotomy $3.45 \%(n=4)$. After a 21-month follow-up, anastomoses were considered good for $61.19 \%(41 / 67)$. Failures were recorded in $16.42 \%(n=11)$ and $22.39 \%(n=15)$ were lost to follow-up. For dilations to the benign patients at 9 months of follow-up, 59\% $(n=13)$ were rated good, and poor in $41 \%(n=9)$ of the cases. Plasty with grafting was successful in $30 \%(n=3)$ of the cases, and $70 \%(n=7)$ were failed. Johanson's urethroplasty was considered satisfactory after six months of recession in $23.07 \%$ (3/13) of the cases, and unsatisfactory in $76.93 \%$ $(10 / 13)$ of the cases. All meatotomies were successful $(n=4)$. An RPM $\leq 30$ $\mathrm{ml}$ was recorded in $55.17 \%$ of the cases and $\geq 100 \mathrm{ml}$ in $31.90 \%$ of the cases. Fifteen patients (12.93) were lost to follow-up after their first evaluation. Conclusion: Stenosis of the urethra is a common condition in consultations at the NHZ. Patients often consult for complications. The UC is the key to the topographic diagnosis of the lesion. Resection with end to end anastomosis gives good results. Plasties with mucosal or skin grafting still remain a challenge in our context.

Keywords: Stenosis, Urethra, Urethroplasty, Surgery, Zinder

\section{Introduction}

La sténose de l'urètre ( $\mathrm{SU}$ ) masculin est une diminution permanente et intrinsèque du calibre de l'urètre constituant à des degrés divers un obstacle à l'écoulement des urines au cours de la miction (Ekeke, 2017 ; Fall, 2018). C'est une pathologie fréquente dont les principales étiologies sont infectieuses, traumatiques ou iatrogènes Wessells, 2017; Fall, 2011). Depuis l'avènement de l'antibiothérapie, la prévalence des étiologies infectieuses est en nette diminution, alors que les causes traumatiques et iatrogènes progressent du fait des accidents de la voie publique et des manœuvres endourologiques (Fall, 2011).

Aujourd'hui, cette pathologie occupe l'actualité chirurgicale à cause de ses complications redoutables, une prise en charge difficile, et les récidives 
fréquentes qui sont à la base de plusieurs recommandations (Oosterlinck, 2015; Wessells, 2017). Au Niger très peu d'études lui sont consacrées. L'objectif de cette étude est de présenter les aspects épidémiologiques, cliniques et thérapeutiques de la sténose urétrale à l'hôpital national de ZINDER (HNZ).

\section{Patients et méthodes :}

Il s'agissait d'une étude rétrospective descriptive réalisée dans le service d'Urologie de l'HNZ sur une période de 4ans (janvier 2016 à Décembre 2019). Les sources de données étaient les dossiers des patients hospitalisés dans ce service, chez qui le diagnostic de rétrécissement urétral était posé, documenté par une UCRM et pris en charge.

Tous les dossiers étaient complets. Les informations recueillies portaient sur l'âge, le motif de consultation, les signes de l'examen physique, les caractéristiques de la sténose sur l'urétro-cystographie rétrograde et mictionnelle (UCRM), l'examen cytobactériologique des urines (ECBU), le type de traitement, et le suivi post opératoire. Les résultats sont évalués le premier mois, le troisième, et le sixième mois sur la qualité du jet urinaire, le résidu post mictionnel (RPM) et la nécessité d'un geste complémentaire comme la dilatation.

Un RPM $\leq 30 \mathrm{ml}$ sans plaintes était considéré satisfaisant et un RPM $\geq 100 \mathrm{ml}$ était non satisfaisant. La nécessité d'un suivi rapproché ou d'une dilatation urétrale était considérée comme des échecs.

Les données étaient enregistrées sur Microsoft Excel 2013 et analysées avec Epi-info 7.

\section{Technique opératoire pour la résection anastomose de l'urètre bulbaire.}

Le patient est installé en position de la taille sous anesthésie locorégionale.

Une asepsie abdomino-périnéale débordant sur les cuisses est faite, puis le champage. Un béniqué $\mathrm{N}^{\circ} 48$ est introduit à travers la cystostomie, et un autre par le méat urétral, ce qui permet de repérer la sténose.

Le scrotum est ensuite tiré vers le haut et fixé aux cuisses pour exposer le périnée. Une incision verticale sur le raphé médian périnéal est faite.

Après les tissus et fascias sous-cutanés, le muscle bulbo-spongieux est exposé puis divisé en deux. Les deux parties de ce muscle sont ensuite écartées latéralement pour exposer l'urètre. L'urètre est disséqué sur toute sa circonférence (figure 1). La partie rétrécie est mesurée avant d'être sectionnée. Les bouts proximal et distal de l'urètre sont spatulés de façon opposée et biseautés aussi de façon opposée de sorte que le biseau proximal s'emboite dans la spatule distale. L'anastomose est faite au tour d'une sonde urétrovésicale gardée pendant deux semaines. 

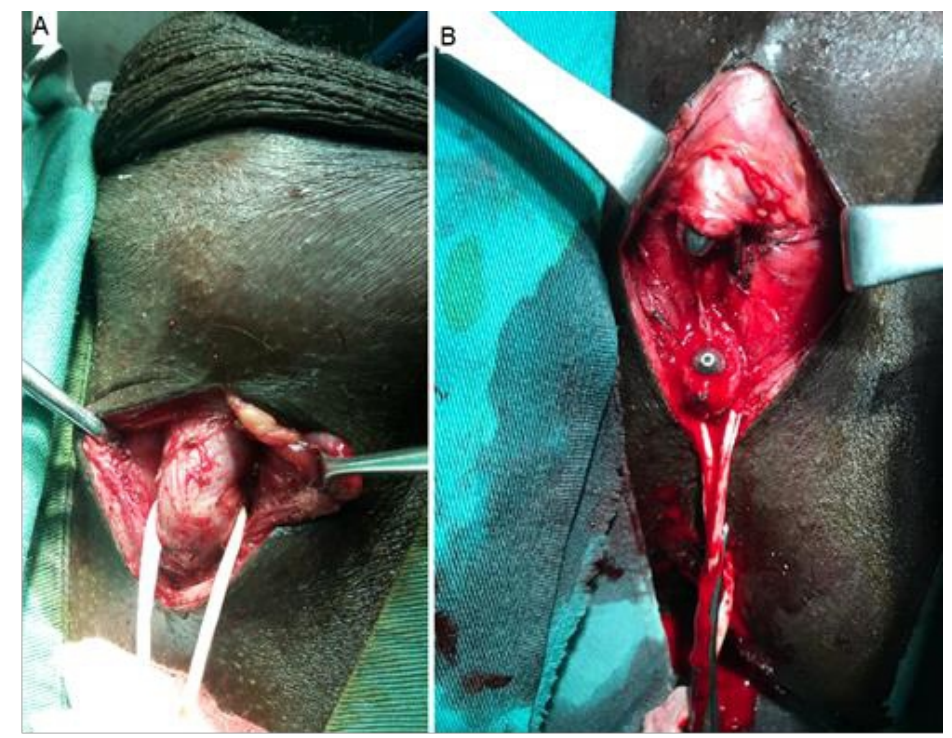

Figure 1 : 1.A.Dissection circonférentielle de l'urètre.1.B.Résection de la sténose

\section{Résultats :}

$\mathrm{Au}$ cours de cette étude, 116 dossiers étaient retenus sur 964 interventions urologiques durant la période, soit une proportion de 8, 31\%.L'âge moyen des patients était de 39,53 ans avec des extrêmes de 18 ans et 74 ans. La tranche d'âge de 18 à 41 ans représentait 60,34\% de l'effectif. Le Tableau I résume la répartition des patients par tranches d'âges. Ces patients provenaient essentiellement de trois régions limitrophes de la région Zinder et d'un pays voisin. Ils étaient à $71,32 \%$ dans des zones rurales et pastorales du nord et de l'est du Niger.

Tableau 1 : Répartition Des Patients Par Tranches D'âges

\begin{tabular}{|c|c|}
\hline Age (ans) & Nombre(\%) \\
\hline$[18-29]$ & $35(30,17)$ \\
\hline$[30-41]$ & $35(30,17)$ \\
\hline$[42-53]$ & $25(21,55)$ \\
\hline$[54-65]$ & $16(13,80)$ \\
\hline$[66-74]$ & $5(4,31)$ \\
\hline TOTAL & $\mathbf{1 1 6}(\mathbf{1 0 0})$ \\
\hline
\end{tabular}

La dysurie était retrouvée dans $25,86 \%$ des cas, une pollakiurie dans 15,52\%, et une rétention vésicale complète d'urine dans $17,24 \%$. Les motifs de consultations à la base de la découverte de la sténose urétrale sont répertoriés dans le Tableau II. 
Tableau II : répartition des patients selon le motif de consultation

\begin{tabular}{|l|c|}
\hline Motifs de Consultations & Nombre (\%) \\
\hline Dysurie & $30(25,86)$ \\
\hline Rétention Vésicale Complète D’urine & $20(17,24)$ \\
\hline Pollakiurie & $18(15,52)$ \\
\hline Gouttes Retardataires & $11(9,48)$ \\
\hline Insuffisance Rénale Aiguë & $8(6,87)$ \\
\hline Orchi-Epididymite Aigue & $8(6,87)$ \\
\hline Algies Pelviennes Chroniques & $8(6,87)$ \\
\hline Edème Des Organes Génitaux Externes & $5(4,31)$ \\
\hline Hernie Inguinale & $4(3,49)$ \\
\hline Fistule Urétro-Cutanée & $4(3,49)$ \\
\hline Total & $\mathbf{1 1 6}(\mathbf{1 0 0})$ \\
\hline
\end{tabular}

L'ECBU avait permis d'isoler E. coli dans 37,06\% $(\mathrm{n}=43)$, klebsiella pneumoniae dans $14,65 \%(n=17)$, staphylococcus aureus dans $8,62 \%(n=10)$ et P. mirabilis dans $2,58 \%(n=3)$. Les autres cultures étaient sans croissance. L'UCRM (figure 2 A et B) était le principal examen complémentaire du diagnostic, il avait permis de découvrir, 63,79\% $(\mathrm{n}=74)$ de sténose urétrale au niveau bulbaire, $18,97 \%(\mathrm{n}=22)$ sténoses péniennes antérieures, $13,79 \%(\mathrm{n}=16)$ sténoses multiples, et 3,45\% $(\mathrm{n}=4)$ au niveau du méat urétral.
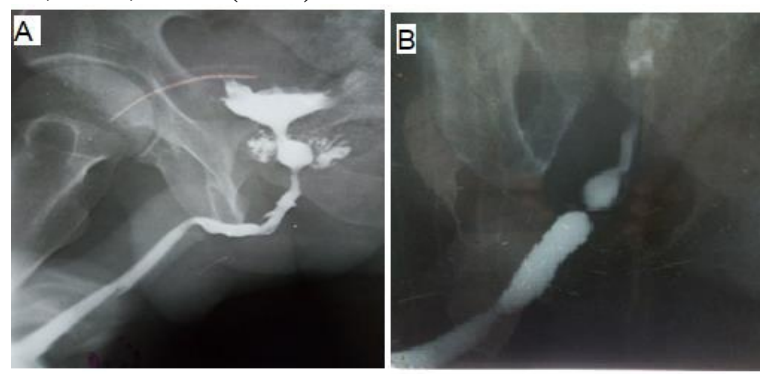

C

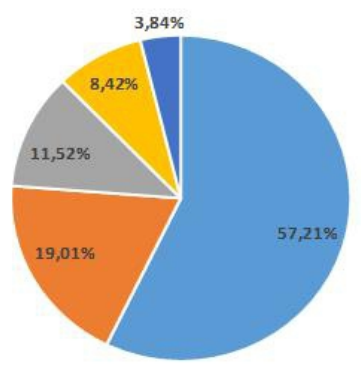

- ANASTOMOSE TT = DILATATION = DEUX TEMPS - GREFFE = MEATOTOMIE

Figure 2 : 2.A. Sténose avec diffusion prostatique du produit de contraste (douleurs pelviennes chroniques)- 2.B. Sténoses multiples- 3.C. Topographie de la sténose sur l'urètre

La longueur moyenne de la sténose était de $1,8 \mathrm{~cm}(0,7-4,5)$

Le traitement avait consisté en une résection anastomose terminoterminale (RATT) au niveau bulbaire dans $57,21 \%(\mathrm{n}=67)$, des cas des 
dilatations urétrales (DU) aux béniqués dans 19, 01\% (n=22) des cas, urétroplastie en deux temps selon Johanson dans $11,52 \%(\mathrm{n}=13)$ et des plasties par greffe de peau glabre de la crête iliaque dans $8,42 \%(n=10)$, une méatotomie $3,45 \%(n=4)$.

Après 21 mois de recul, les anastomoses (RATT) étaient jugées bonnes pour $61,19 \%$ (41/67). Les échecs étaient enregistrés dans 16,42\% (n=11) et $22,39 \%(n=15)$ étaient perdus de vue. Pour les dilatations aux béniqués à 9 mois de recul, 59\% ( $n=13)$ étaient évalués bons, et mauvais dans $41 \%(n=9)$ des cas. Les plasties avec greffe étaient réussies dans $30 \%(n=3)$ des cas, et $70 \%(\mathrm{n}=7)$ étaient des échecs. Les urétroplasties selon Johanson étaient jugées satisfaisantes à six mois de récul dans 23,07\% (3/13) des cas, et non satisfaisants dans 76,93\% (10/13) des cas.Toutes les méatotomies étaient réussies $(\mathrm{n}=4) . \quad$ Un $R P M \leq 30 \mathrm{ml}$ était enregistré dans $55,17 \%$ des cas et $\geq$ $100 \mathrm{ml}$ dans $31,90 \%$ des cas. Quinze patients soit 12,93 étaient perdus de vue après leur première évaluation.

\section{Discussion}

Sur un total de 964 interventions en urologie durant la période, 116 étaient des sténoses urétrales soit une proportion de $8,31 \%$. Nos résultats confirment la tendance générale dans la sous-région, où, au Bénin, au BurkinaFaso, et au Mali elle avait une prévalence hospitalière respectivement de 11,3\%, 6,2\%, et 7,14\% (Hounnasso, 2015; Kaboré, 2015; Salifou, 2019), mais largement supérieur à $0.82 \%$ de Kouamé et coll à Dakar (Kouamé, 2017).

C'est une pathologie de l'adulte jeune en Afrique, dans la majorité des cas entre 40 à 55 ans (Ekeke, 2017 ; Fall, 2018 ;Hounnasso, 2015 ; Fall, 2011 ; Kouamé 2017 ; Moby 2018).

L'âge moyen de nos patients était de 39,53ans avec des extrêmes de 18 et 74 ans. Il se situe dans la moyenne générale d'Afrique au sud du Sahara où les patients appartenaient dans $80 \%$ des cas à la tranche d'âge de 16-50ans (Ekeke, 2017; Fall, 2011 ;Ndoye, 2016 ; Kaboré, 2015; Ouattara, 2004). Par contre dans les pays développés la moyenne d'âge commence à partir de 55 ans (Lazzeri, 2016; Verla 2019), période pendant laquelle débutent les troubles urinaires liés aux pathologies prostatiques et les manœuvres endourologiques.

Ces manœuvres, même mini invasives, comme le cathétérisme urétral sont reconnues pourvoyeuses de sténose urétrale (Ondo, 2015; Verla, 2019).

La sténose de l'urètre (SU) est une pathologie dont les principales étiologies varient d'une région à une autre. Dans les pays développés et en voie de développement, les formes idiopathiques et iatrogéniques sont majoritaires (Fall, 2011; Lazzeri, 2016; Tritschler, 2013; Stein, 2013), à l'opposée en Afrique subsaharienne depuis les années 1980, 50-80\% des sténoses urétrales étaient dues à des infections du tractus urinaire répétées et mal traitées (Fall, 2011 ; Ndoye, 2016 ). Cependant Ekeke, rapportait une part 
plus importante de causes traumatiques dans sa série notamment les traumatismes périnéaux par le bord de la pirogue chez les pêcheurs (Ekeke, 2017 ), de même, Viers attirait l'attention sur les traumatismes minimes, mais répétés avant toute déduction sur le caractère idiopathique de la sténose(Viers, 2017 ).cette particularité semble rejoindre notre contexte, où une autre donnée spécifique de certaines zones nomades n'est pas négligeable, notamment les traumatismes minimes, mais répétés chez les jeunes nomades à dos d'âne nu.

Cette entité doit être considérée d'autant plus que 19 patients soit $16,37 \%$ avaient les mêmes habitudes pastorales et étaient arrivés avec une sténose bulbaire et une hyperkératose périnéale témoin de ces microtraumatismes.

Les motifs de consultations à la base de la découverte de sténose urétrale étaient principalement les troubles mictionnels du bas appareil urinaires dont la dysurie représentait $(25,86 \%)$ dans notre étude. Elle était la principale circonstance de découverte de la sténose urétrale dans respectivement $100 \%, 70,17 \%$ et $67,6 \%$ pour Ondo au Sénégal,Ngaroua au Cameroun, et Sikpa au Togo (Ondo, 2015; Ngaroua, 2017; Sikpa, 2016).

La dysurie s'installe de façon insidieuse laissant une marge d'adaptation au patient ce qui favorise le retard de consultation et le développement des complications.

Les complications représentaient $57,8 \%$ des circonstances de découverte de la pathologie pour Fall (Fall, 2011).

Au-delà de ces complications fréquentes, nous avons enregistré, 6,87\% d'insuffisants rénaux aiguë référés pour une prise en charge de la pathologie obstructive avec une crétininémie variant entre 157 à $632 \mu \mathrm{mol} / \mathrm{L}$, et plusieurs cas d'orchiépididymite et d'algies pelviennes chroniques dont le diagnostic étiologique avait abouti à la sténose urétrale.

Sur le plan paraclinique L'UCRM était le principal examen complémentaire du diagnostic, il avait permis de découvrir, 63,79\%(n=74) de sténose au niveau bulbaire notre taux est proche de ceux de Sikpa et Ndour avec respectivement 67,6\% et 67,8\%, ( Sikpa, 2016; N'Dour, 2018).

L'ECBU avait permis d'isoler des germes dans certains cas, qui tous présentaient une grande résistance aux amino-pénicillines.

Le traitement de la sténose urétrale à évoluer passant de la dilatation à l'urethrotomie interne et à la plastie. Les indications de la technique opératoire sont de plus en plus précises (Oosterlinck, 2015; Wessels, 2017; Hussain, 2020; Daneshvar, 2020; Buckley, 2014).

Les résultats des procédures dépendent dans tous les cas d'une bonne analyse des facteurs comme; l'étiologie, la localisation, la longueur, la densité, les gestes antérieurs, les facteurs de comorbidité, et surtout de l'expérience du chirurgien dans l'évaluation de ces facteurs pour le choix de 
la procédure (Buckley, 2014; Jarry, 2017; Gimbernat, 2014; Barbagli, 2014; Bradley, 2017; Robine, 2017).

La résection anastomose termino-terminale (RATT) est considérée comme le traitement de première intention pour les sténoses courtes au niveau bulbaire. Cette technique est décrite par la majorité des auteurs comme étant accessible et donnant des bons résultats allant de 62,5\% à 98,8\% selon les études (Salifou, 2019; Hussain, 2020; Robine, 2017). Nous avions procédé à $67(57,21 \%)$ RATT, parmi lesquels $41 / 67$ soit $61,19 \%$ sont jugés bons avec 21 mois de recul, $11(16,42 \%)$ étaient des échecs, et 15 (22,39\%.) étaient perdus de vue.

La dilatation au béniqué était la première procédure du traitement de la sténose urétrale, ses résultats sont controversés, car grevés de récidive à court terme. Elle reste encore pratiquée souvent en première intention, par Hussain dans 33,7\% (Hussain, 2020), et Salifou dans 16,66\% (Salifou, 2019). Nous avions procédé à des dilatations aux béniqués chez 22 patients, dont 13 étaient évalués bons à 9 mois et 9 échecs.

Les plasties et greffes représentent le gold standard du traitement des sténoses étendues. Elles nécessitent une grande expérience du chirurgien et un bon plateau technique. Dans notre contexte ce sont les infections post opératoires et les nécroses du greffon qui étaient à la base des échecs. Nous avions enregistré 3 plasties avec greffe jugées bonnes et 7 échecs.

\section{Conclusion}

La sténose de l'urètre est une affection fréquente dans les consultations à l'HNZ. Les patients consultent souvent pour les complications. L'UCRM est la clé du diagnostic topographique de la lésion. La résection anastomose termino-terminale donne de bons résultats. La maitrise des plasties avec greffe de muqueuse, ou cutanée reste encore un défi dans notre contexte.

\section{References :}

1. Ekeke O.N. , Amusan O.E (2017). Clinical presentation and treatment of urethral stricture: Experience from a tertiary hospital in Port Harcourt, Nigeria. African Journal of Urology 23: 72-77.

2. Fall B, C. Zeondo, Y. Sow, A. Sarr, B. Sine, A. Thiam, et al (2018). Résultats de l'urétroplastie anastomotique pour rétrécissement de l'urètre masculin. Prog Urol 28 (7): 377-381.

3. Wessells H, Angermeier KW, Elliott S, Christopher M. Gonzalez, Ron Kodama, Andrew C. Peterson et al (2017). Male Urethral Stricture: American Urological Association Guideline. J Urol. 197(1):182-190

4. Boubacar Fall, Yaya Sow, Ismael Mansouri, Alioune Sarr, Amath Thiam, Babacar Diao,et al (2011). Etiology and current clinical 
characteristics of male urethral stricture disease: experience from a public teaching hospital in Senegal. Int Urol Nephrol 43:969-974 DOI 10.1007/s11255-011-9940-y.

5. Oosterlinck W, Lumen N, Van Cauwenberghe G (2015). Traitement chirurgical des sténoses de l'urètre: aspects techniques. Annales d'Urologie 41: 173-207.

6. Hounnasso, P.P., Tore Sanni, R., Avakoudjo, J.D.G., et al. (2015) Aspects épidémiologiques et diagnostiques du rétrécissement de l'urètre masculin au centre national hospitalier universitaire Hubert Koutoukou Maga de Cotonou. Uro'Andro 1: 162-165.

7. Fa Kabore, B Zango, A K Paré, B Kirakoya, T Kambou, A Ouattara et al (2015). Analyse rétrospective du traitement chirurgical d'une série de 148 cas de rétrécissements de l'urètre masculin au Burkina Faso Uro-Andro 1 (4) :200-205.

8. Salifou Issiaka Traore, Ousmane Dembélé, Amadou Maiga, Soumaila Traore, Aly Boubacar Diallo, Toure layes et al (2019).Prise en charge du rétrécissement urétral acquis: expérience du Service de Chirurgie Générale de Sikasso. Pan African Medical Journal 33:328. doi:10.11604/pamj.2019.33.328.16724.

9. B. Kouame, J. Horace, M. Ndoye, F. Kramo, M. Roua, J. J. Gandonou, et al (2017). Men Urethra Strictures: Findings in Urethroplasties Care at the Andrology and Urology Department of Grand Yoff General Hospital in Dakar. Open Journal of Urology 7: 173185http://www.scirp.org/journal/oju ISSN Online: 2160-5629 ISSN Print: 2160-5440.

10. E.H. Moby, S Makon, D Eyongueta, C Kamadjou, B.T. Sala, S Happy, et al (2018). Traitement des sténoses de l'urètre par urétrotomie interne endoscopique : profil épidémiologique, clinique, et thérapeutique à DoualaVol 8 ( 1 ) : 775-783. ISSN: 2410-8936.

11. Ndoye, M., Niang, L., Labou, I., Jalloh, M., \& Gueye, S. (2016). Sténoses de l'urètre au service d'urologie de l'hôpital général de Grand Yoff. Résultats des urétroplasties de 2001 à 2013. Progrès En Urologie 26(13): 745. doi:10.1016/j.purol.2016.07.158.

12. Ouattara Z, Tembely A, Sanogo Zz, Doumbia D, Cisse Cmc, Ouattara $\mathrm{K}$ et al (2004). Rétrécissement De L'urètre Chez L'homme A L'hôpital Du Point « G ». Mali Médical T XIX ( 3\&4): 48-50.

13. Massimo Lazzeri, Salvatore Sansalone, Giorgio Guazzoni, Guido Barbagli et al (2016). Incidence, Causes, and Complications of Urethral Stricture DiseaseEuropean urology supplements 15: 2-6.

14. Wesley Verla, Willem Oosterlinck, Anne-Françoise Spinoit, MarjanWaterloos (2019). A Comprehensive Review Emphasizing Anatomy, Etiology, Diagnosis, and Treatment of Male Urethral 
Stricture Disease. BioMed Research International Volume 2019: 1-20. Article ID 9046430, https://doi.org/10.1155/2019/9046430.

15. C.Ze. Ondo, B. Fall, Y. Diallo , Y. Sowa, A. Sarr, R. Ngonga et al (2015). Les rétrécissements iatrogènes de l'urètre: expérience d'un hôpital Sénégalais. African journal of urology 21: 144-147.

16. Stefan Tritschler, Alexander Roosen, Claudius Füllhase, Christian G. Stief, Herbert Rübben (2013). Urethral Stricture: Etiology, Investigation and Treatments. Deutsches Arzteblatt International 110: 220-22.

17. D. M. Stein, D. J. Thum, G. Barbagli et al (2013). A geographic analysis of male urethral stricture aetiology and location,BJU International. vol. 112 (6): 830-834.

18. Boyd R. Viers, Travis J. Pagliara, Charles A. Rew, Lauren Folgosa Cooley, Christine Y. Shiang, Jeremy M et al (2017). Characteristics of Idiopathic Urethral Strictures: a Link to Remote Perineal Trauma?Urology $2017 \quad$ : 20. http://dx.doi.org/doi:10.1016/j.urology.2017.07.022.

19. Ngaroua, Ngah Joseph Eloundou, Yaouba Djibrilla, Ousmane Asmaou, Amvene Jérémie Mbo (2017). Aspects épidémiologiques, cliniques et prise en charge de sténose urétrale chez l'adulte dans un Hôpital de District de Ngaoundéré, Cameroun. Pan African Medical Journal 26; 193. doi:10.11604/pamj.2017.26.193.9669.

20. K.H.SIKPA, T.M. Kpatcha, K.K. Tengue, E.V. Sewa, G. Botcho, F. Soumanou et al (2016). L'Urétroplastie par résection anastomose termino-terminale pour rétrécissement del'urètre masculin au CHU Sylvanus Olympio de Lomé au Togo. African journal of urology 22: 273-278.

21. N. S. Ndour, I. D. Diamé, Y.Diallo, S C. Kouka, M. Ndiaye, I. Diallo, et al. Prise en charge du rétrécissement de l'urètre : Notre expérience en milieu rural.Uro'Andro $2018 ; 1 ; 11,528-32$.

22. Hussain M, Khan MS, Lal M, Hashmi A, Naqvi SAA, Rizvi SAH(2020). Stricture of urethra: Patterns and outcomes of management from a single centre in Pakistan over 7 years. J Coll Physicians Surg Pak 30(1):79-84.

23. Michael Daneshvar, Jay Simhan, Stephen Blakely, Javier C. Angulo, Jacob Lucas, Craig Hunter, et al (2020). Transurethral ventral buccal mucosa graft inlay for treatment of distal urethral strictures: international multi-institutional experience. World Journal of Urology janvier 2020 https://doi.org/10.1007/s00345-019-03061-6.

24. Buckley JC, Heyns C, Gilling P, Carney J et al (2014). consultation on urethral strictures: dilation, internal urethrotomy, andstenting of male anterior urethral strictures. Urology 83(3):18-22. 
25. L. Jarry, V. Ravery, A. Daché, J.-F. Hermieu, C. Egrot, I. Ouzaid (2017)L'urétroplastie par résection anastomose en un temps : impact du mécanisme lésionnel sur la prise en charge et les résultats. Progrès en urologie 27: 184-189.

26. Gimbernat H, Arance I, Redondo C, et al (2014). Analysis of the factors involved in the failure of urethroplasty in men. Actas Urol Esp. 38(2):96-102.

27. Barbagli G, Kulkarni SB, Fossati N, et al (2014). Long-term followup and deterioration rate of anterior substitution urethroplasty. J Urol. 192(3):808-813.

28. Bradley A. Erickson, MD, MS, George M. Ghareeb, MD, MBA (2017).Definition of Successful Treatment and Optimal Follow-up after Urethral Reconstruction for Urethral Stricture Disease.Urol Clin N Am 44 : 1-9 http://dx.doi.org/10.1016/j.ucl.2016.08.001

29. E. Robine, J. Rigaud, F. Luyckx, Q.-C. Le Clerc, F.-X. Madec, O. Bouchot, J. Branchereau (2017). Analyse des taux de succès des urétroplasties pour sténoses de l'urètre bulbaire chez l'homme adulte : revue systématique de la littérature. Progrès en urologie 27: 49—57 